\title{
ASTHMA DYNAMICS AND MEDICAL AMELIORATION
}

\author{
Dr. Meyer Katzper \\ Center for Drug Evaluation and Research, FDA \\ 5600 Fishers Lane \\ Rockville, Maryland 20857
}

\begin{abstract}
Asthma and chronic obstructive pulmonary disease impede airflow in the lung. Various medications are used to ameliorate asthmatic attacks. The use of bronchodilators has been studied and data on dose-response analyzed. The effects seen in cumulative dosing are shown to fit an Emax model. Values obtained assist in the determination of dosing levels. Simulation studies are used here to estimate the relationship of doses to concentration levels and the differential effects of increased drug levels. A visually oriented platform, STELLA, is used for developing and exploring pharmacodynamic models of lung function. Examination of the consequences of alternate pharmacodynamic model formulations provides guidance for improved dosing. The simulations carried out were designed to capture the dynamic time course of recovery from an asthma attack. They demonstrate when cumulative doses should be avoided.
\end{abstract}

\section{INTRODUCTION}

Asthma is characterized by episodes of breathing difficulty commonly referred to as "attacks." These attacks are due to widespread narrowing of the airways of the lung. It is not generally recognized by the public that these attacks can be life threatening. The more general category of chronic pulmonary obstructive disease, which includes adult asthma, is estimated to be the fifth leading cause of death in the U.S. (Boyars 1988). Overmedication itself may lead to adverse clinical effects. Our study focused on the course of an attack with different bronchodilator doses.

\section{BACKGROUND}

Narrowing of the airways in asthma may be due to mucus in the airway, swelling of the lining in the airway, spasm of the muscle in the airway or all of these. This is shown schematically in Figure 1 (adapted from Lane and Stor, 1979). Data we have looked at comes from patients with episodes of bronchoconstriction not involving mucus. In normal individuals this parasympathetic reflex action is designed to expel or prevent entry of foreign matter to the lungs. The asthmatic is oversensitized and overresponds with bronchial spasm.

Rapid relief is available by inhalation of a bronchodilator from a metered dose inhaler. Most frequently the active ingredient is a beta-2-adrenergic agonist which relaxes the bronchial muscles.

Lung function is measured with spirometers which record expired volume versus time. A reliable measure of bronchodilator effect is the increase in forced expiratory volume in one second, FEV 1 (Light, Conrad and George, 1977, Berger and Smith, 1988). The data we have studied has been cumulative-dose response of FEV 1 to albuterol (Barnes and Pride, 1983, Hendeles et. al., 1992).

Concentration determinations form a basis for setting dosing levels for medications. Pharmacokinetic parameters obtained by fitting these models to data provide necessary dosing guidance. It is known in the case of inhalation of a bronchodilator using a metered dose inhaler that systemic plasma concentrations do not correlate well with bronchodilating effect (Walker, Evans, Richards and Patterson, 1972).

We therefore use dosing levels rather than concentration measures. This is very reasonable as the effect in the lung is through the drug concentration in the airways rather than in the plasma. Ideally one would have the time course of concentration in the lung. While lung concentrations are not available, the simulation using dosing levels and dose clearance approximates the dynamics of drug concentration at receptor sites.

Many conditions are self limiting and the body recovers without medical intervention. The dynamics of recovery with and without intervention are studied here. 


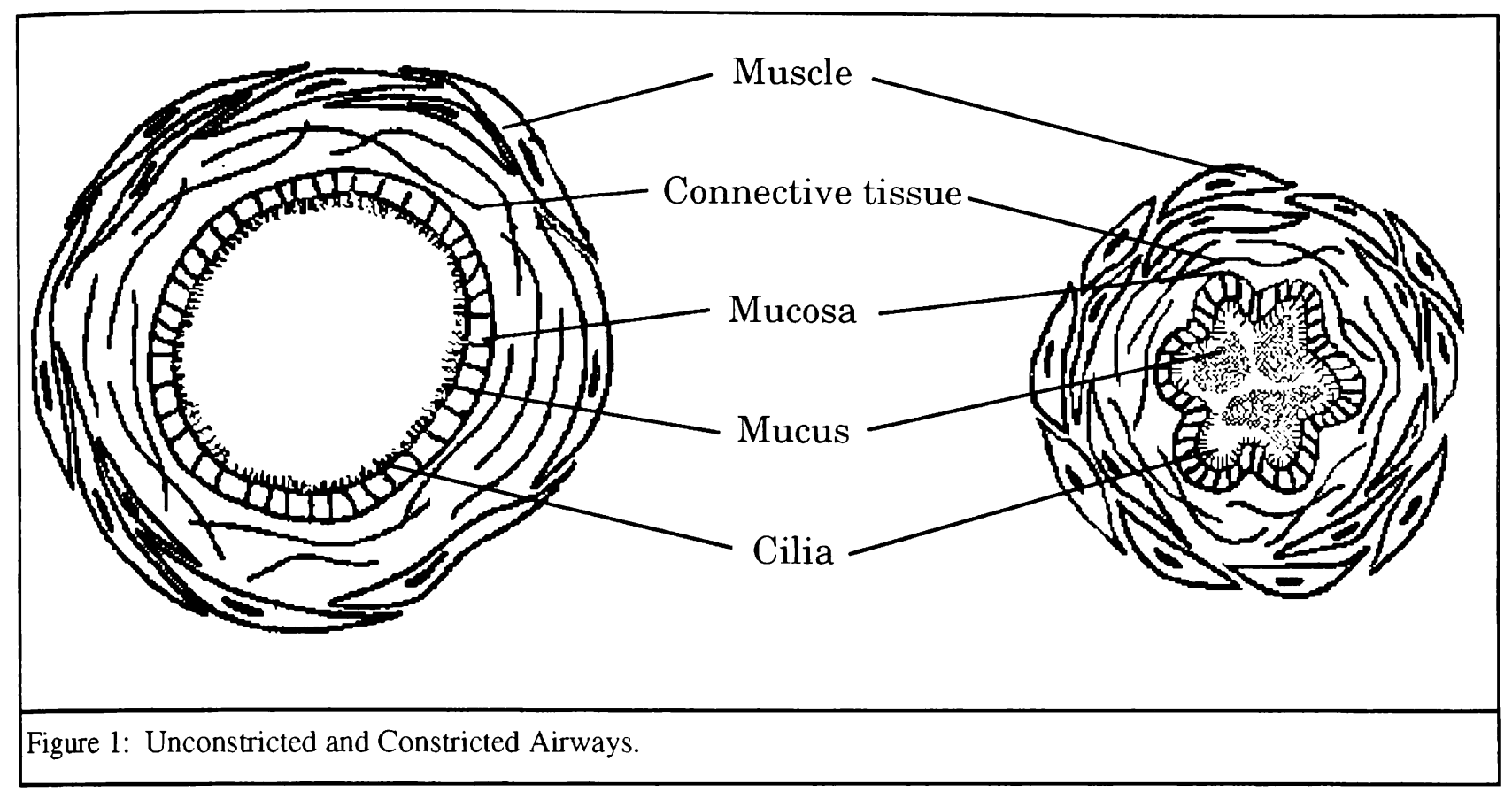

\section{MODELING CONSIDERATIONS}

As in most modeling, major simplifications underlie our analysis. For many drugs, effect data is fitted to simple descriptive phenomenological equations. An equation frequently used when larger doses have smaller incremental effects approaching a limiting maximum value is the Emax model.

The Emax model equation is:

$$
\text { Effect }=\mathrm{E} 0+\frac{\text { Emax } * \text { Dose }}{\text { ED50 }+ \text { Dose }}
$$

where $\mathrm{E} 0$ is the baseline value, Emax is the maximum effect from baseline, and ED50 creates half of the maximum effect. When the Emax model is appropriate, these parameters characterize the dose-response relationship.

We seek to proceed beyond model curve fitting to understand the response of the lung from a physiologically relevant control viewpoint.

The lung may be modeled by a limited number of interacting compartments. A complex, continuous and largely nonhomogeneous system is replaced by a discrete number of homogeneous compartments and controls. The dynamics of such a compartmental system can be formulated in terms of sets of differential equations. Compartmental analysis is a standard approach in pharmacokinetics.
Here an equivalent visually programmed representation of the compartments and rate controllers will be used for model formulation and simulation.

\section{APPLYING THE EMAX MODEL}

Data for each individual was fit to an Emax model to get parametric characterizations, a measure of interpatient variability and a comparative measure of dose effect.

Data were fitted from literature values (Barnes and Pride 1983) and from data obtained through Dr. Leslie Hendeles from a clinical study of asthmatic attacks and medication both during the day and during the night. In Figure 2 we show a sample Emax model fit. The fits generally tracked the data well. Average nightime E0 was lower and nighttime Emax and ED50 higher.

The effects seen in this and other cumulative dosing studies are fit in such a manner as to attribute the higher effect to the total dosing at the time of effect. The observed effect can be a result of continuing relaxation due to earlier doses and additional relaxation due to the increased drug concentration.

But the earlier doses have at least partially been eliminated so that an addition of doses may not be warranted. Furthermore, it is likely that the drug-assisted underlying control mechanism may continue to drive the system toward equilibrium in the absence of additional doses. We therefore formulated a model to simulate the dynamics of response in the presence of a control mechanism. 


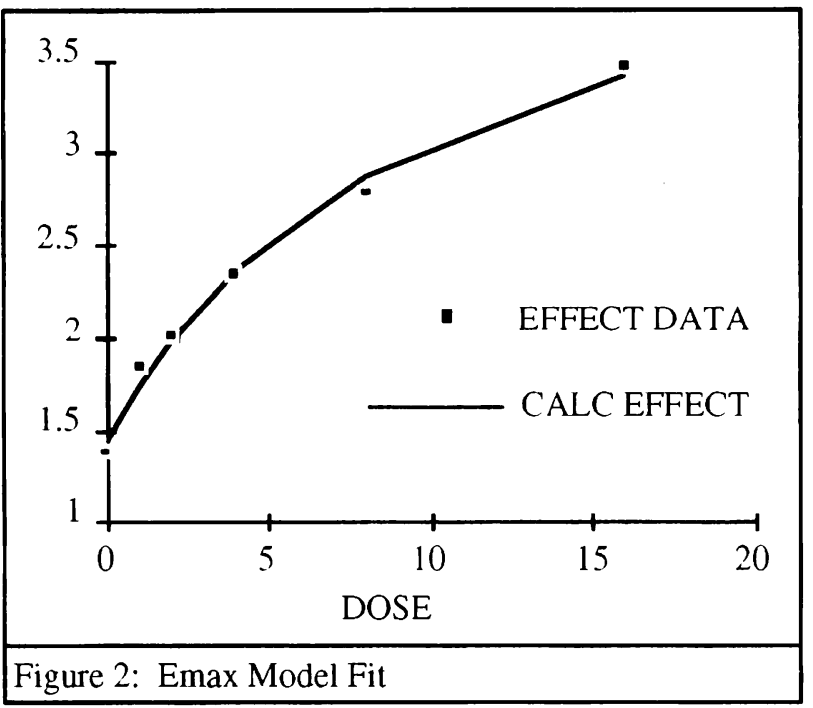

\section{SOFTWARE USED}

The software used for the dynamic simulations is STELLA (Bogen, 1989). It operates on the Macintosh computer with a visual formulation of the model. The diagrams are a representation of sets of difference and differential equations. Ease of model formulation and of interactive parameter value exploration make it excellent for exploratory model studies.

There are only four substantive modeling elements in STELLA. All are shown and described in Figure 3 below.

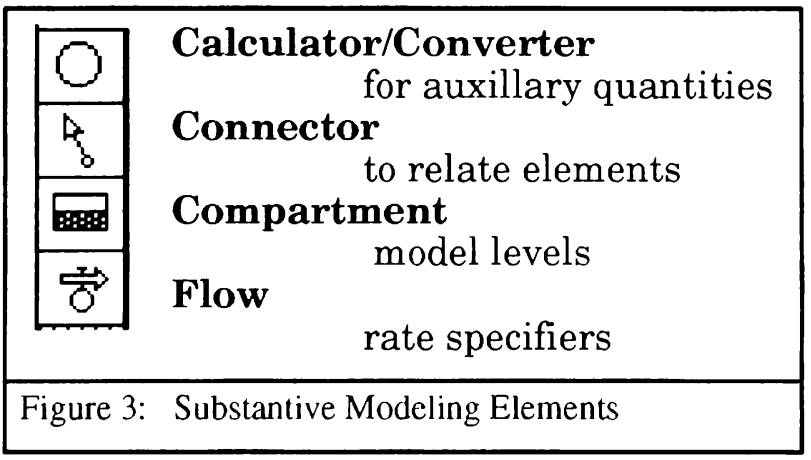

Using these elements in the construction of a diagram is equivalent to writing a set of ordinary differential or difference equations representing the levels of substances in the compartments and the flux between compartments.

As an example of use of STELLA, consider an initial amount of substance undergoing decay or elimination in proportion to the amount of substance. This is expressed by the differential equation

$\mathrm{dY} / \mathrm{dt}=-\mathrm{kY}$
The STELLA representation, which yields the exponential decay solution is shown in Figure 4. The calculational equation for $\mathrm{Y}$ gets written automatically from the graph as

$\mathrm{Y}(\mathrm{t})=\mathrm{Y}(\mathrm{t}-\mathrm{dt})+(-$ DecayRate $) * d t$

Decay Rate must be assigned subject to the specifications of the connectors. Here

DecayRate $=k * Y$

The model will not run until an initial value for $\mathrm{Y}$, INITY, is assigned.

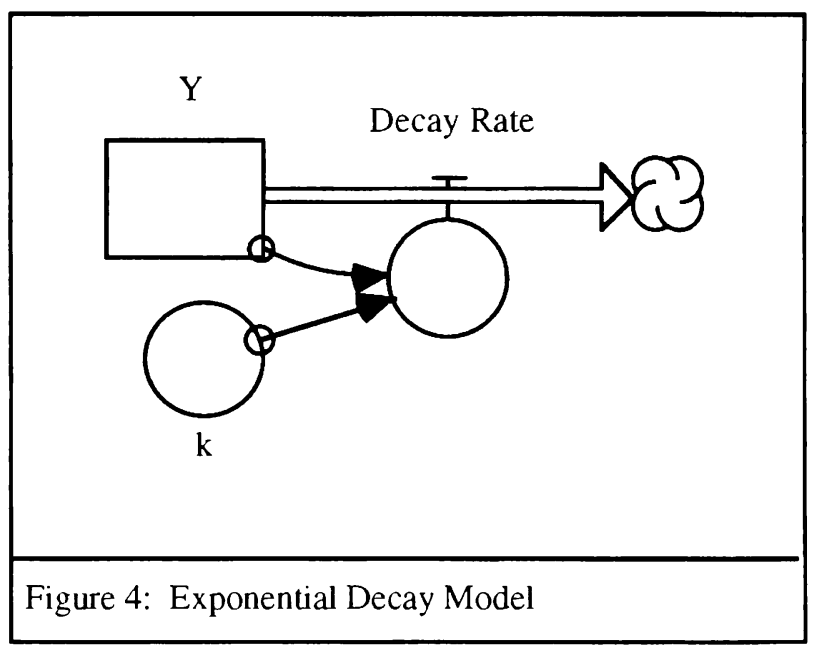

\section{MODEL FORMULATION}

In the healthy, normal individual, there is a balance between the parasympathetic and sympathetic nervous system influences. This balance between constriction and relaxation maintains the proper bronchial smooth muscle tone and airway calibre for ease of airflow. For modeling simplicity (and in the absence of detailed experimental data), these controls will be represented by a classical feedback loop. However, instead of creating a model at the theoretical unobservable level, the model will be formulated in terms of the experimentally determined FEV1. This model is shown in Figure 5. Our visually programmed representation consists of the $\mathrm{FEV}$ rectangle whose change with time is dependent on the rate controlling connecting pipes - Maintenance and Turnover. Rate controlling external connections, such as to dosing or elimination, have little cloud shapes as terminators. The figure also indicates how stress or an attack might affect Turnover and reduce FEV1. 
The left side of the figure embodies the mathematics for the feedback loop. In Maintenance, FEV 1 is continually compaired to the Setpoint value. If FEV 1 falls below the Setpoint, then Maintenance is increased at a rate determined by the Relaxation time.

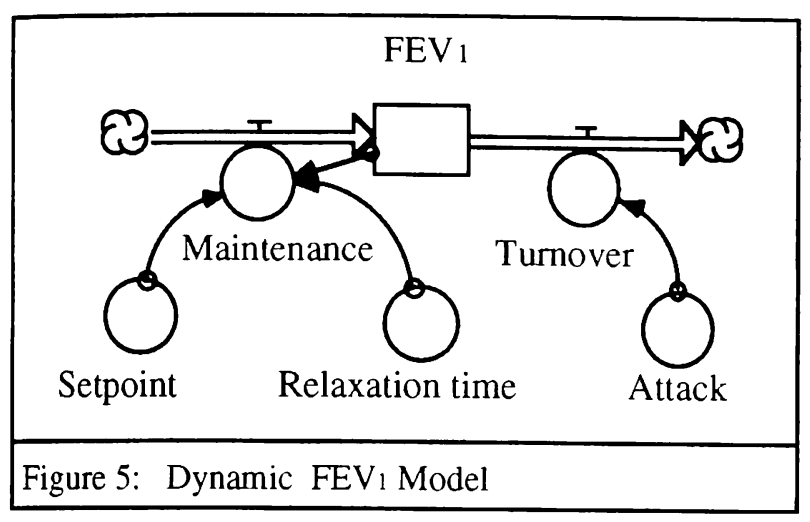

For studying an asthmatic taking medication to alleviate an attack, the pharmacokinetic aspect of the model is linked to the control machinery as shown in Figure 6. The pharmacokinetic model can be formulated and run independently. Drug level will depend on dosing and clearance. Effectiveness depends on receptor sensitivity and the effector mechanism. To account for this a Drug Effect parameter is included in the link of Drug Level to Maintenance.

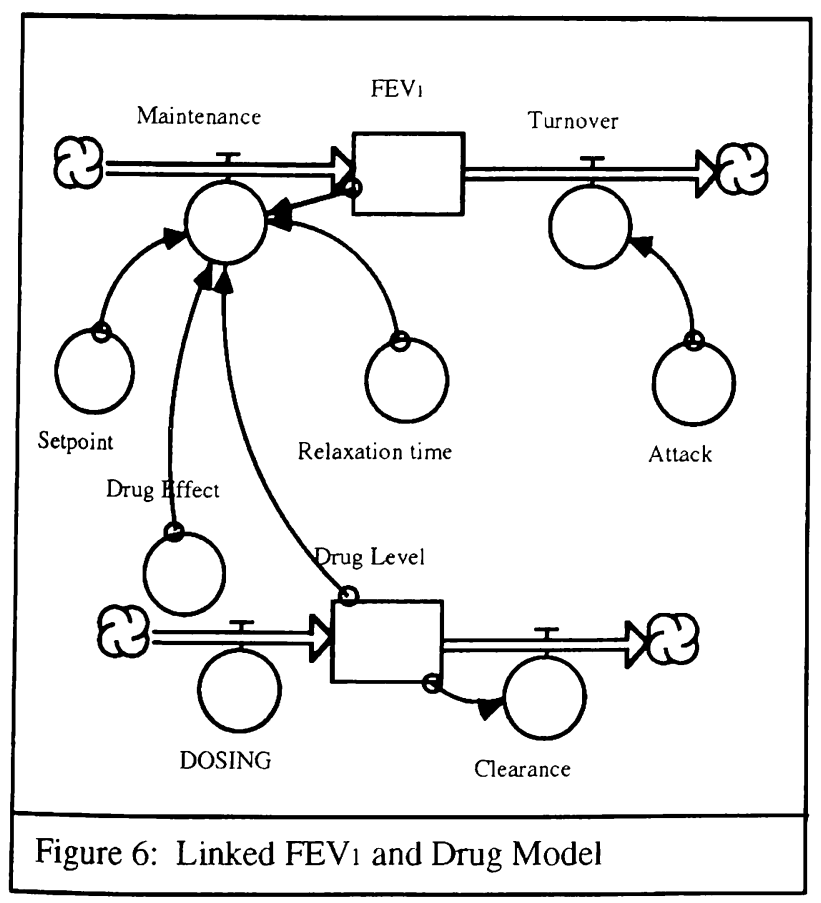

\section{SCENARIOS}

Our basic model can be easily modified to incorporate structurally different scenarios. Consider the effect of exercise. The model is shown in Figure 7a. The feedback mechanism is actually exactly the same as in Figure 5. However, it is now contained in the Maintenance \&response rate for visual clarity. Exercise increases breathing capacity by widening the airways. After exercise the normal person rapidly reequilibrates to the resting state after a small degree of overshoot. However in the asthmatic the decrease in airway calibre may be $20 \%$ and persist two to three hours. A simulation of this is shown in Figure $7 \mathrm{~b}$.

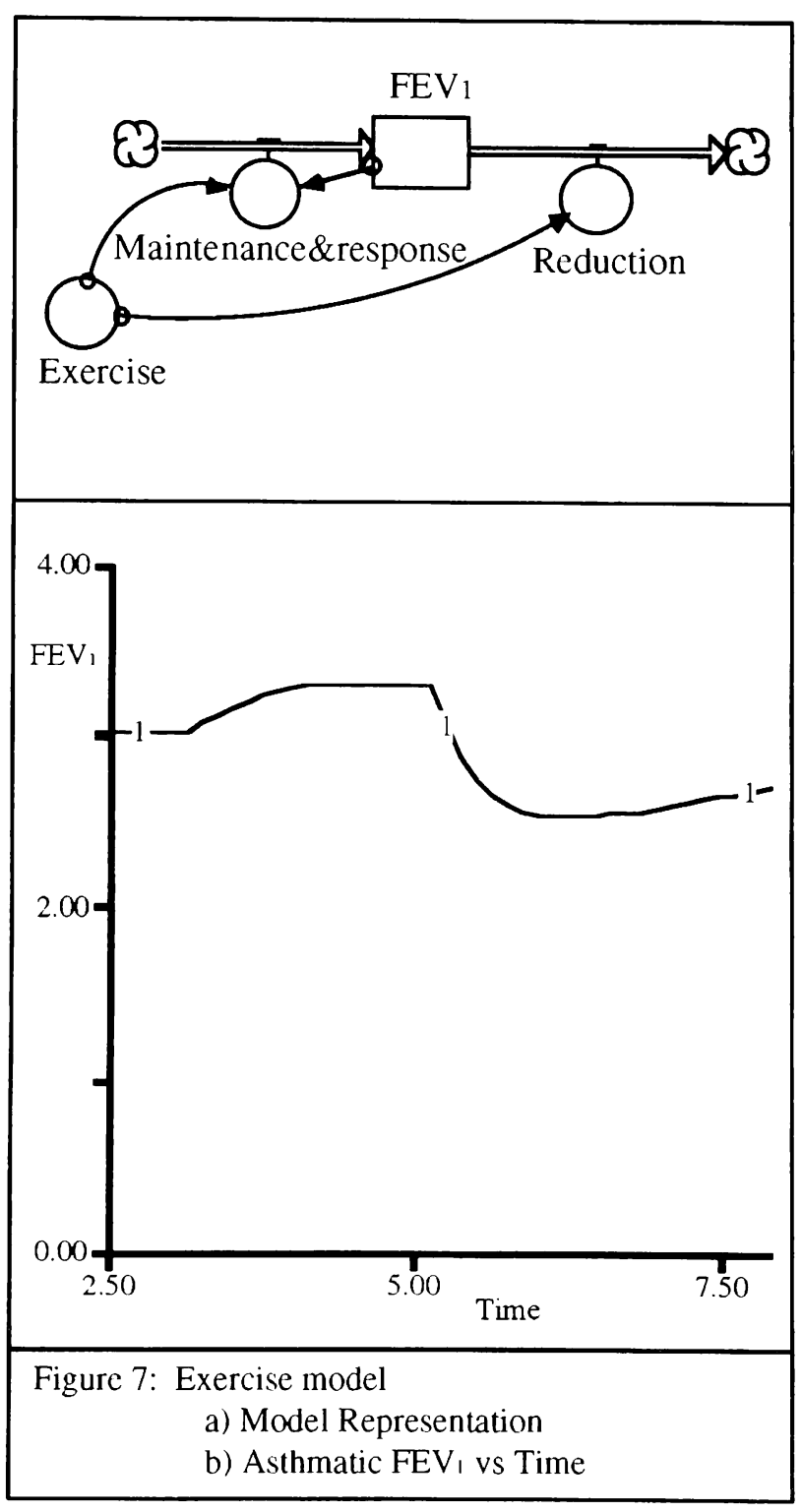


Based on the known effectiveness of bronchodilators and the difficulties of an asthmatic after exercise, it is reasonable to assume that the control mechanisms in an asthmatic are operational, but defective. The asthmatic is hypersensitive to an allergen or exercise or other stimuli. His bronchi overreact and go into spasm in place of achieving ordinary equilibrium. With no medication, relief will be slower. ( We will not deal here with chronic and unrelieved cases.) The bronchi dilate slowly. The bronchodilator speeds up the smooth muscle relaxation by activating the receptors.

Now consider an asthmatic attack which occurs at night. FEV 1 decreases at night and an attack is triggered at midnight. Curve 1 is the recuperation without a bronchodilator and Curve 2 shows the results of a single dose of bronchodilator. The second figure shows the same thing but for multiple doses for Curve 2 .

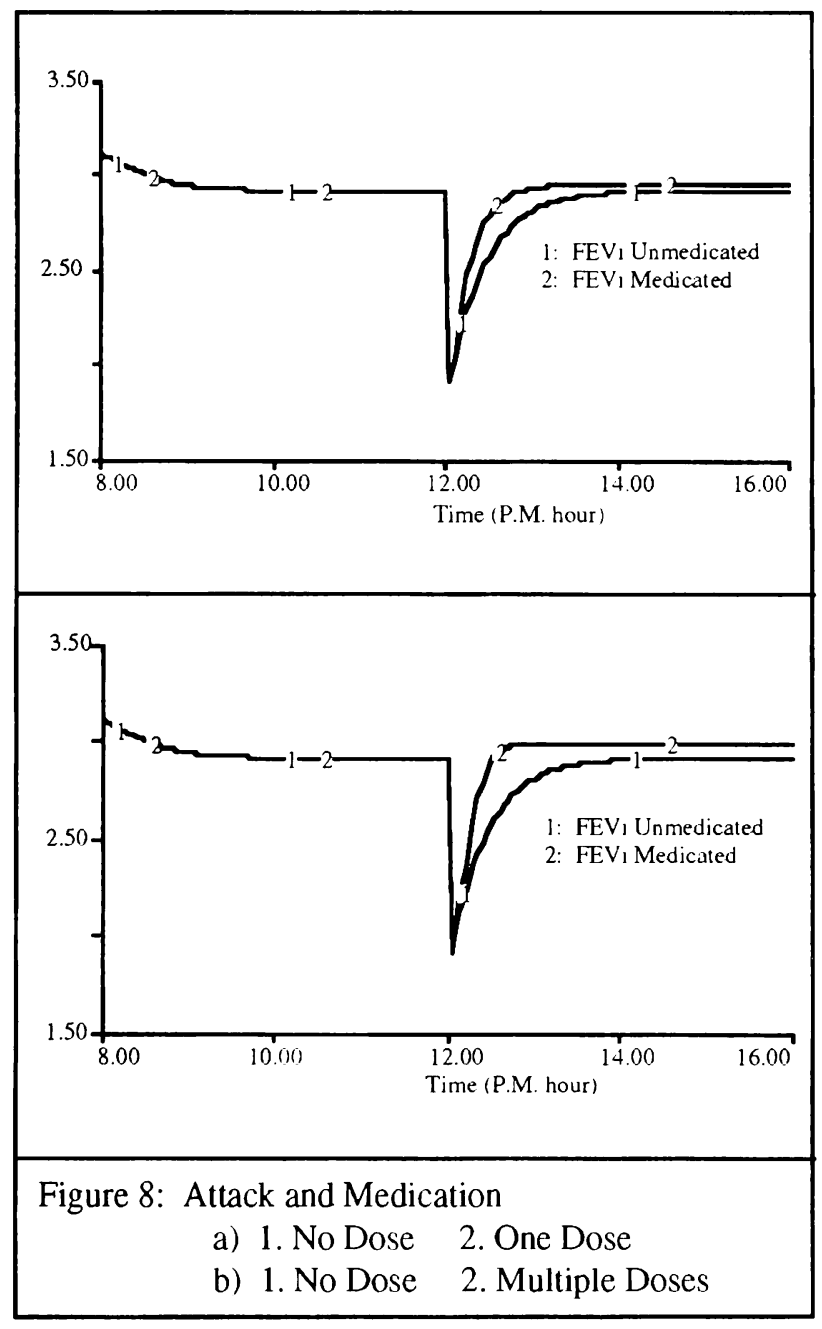

Higher dosing brings quicker, but not much more, relief. It can be seen that a series of doses taken every 15 minutes provides faster and greater relief. However, there is a high cost to this differential in relief. Curve 2 in Figure 9 shows the drug level under multiple dosing with each jag in the curve another dose. It is a natural response for an asthmatic who wakes up in the middle of the night unable to breathe to give not one but a series of puffs from the bronchodilator to obtain relief. Deaths do occur in asthmatics apparently from overdosing. It is clear from Curve 3 of the graph which represents the effects of the control system on FEV 1 that the maximal adjustment occurs with the first three puffs. Subsequent dosing is ineffective.

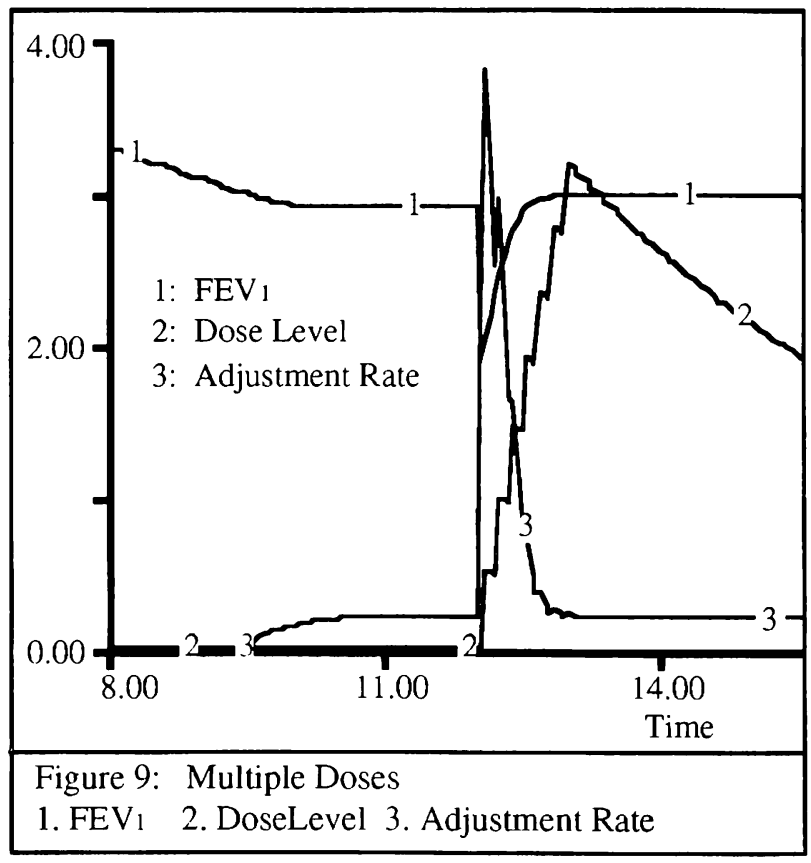

\section{CONCLUSION}

Examination of the consequences of alternate pharmacodynamic model formulations was shown to provide guidance for improved dosing. Data from cumulative dose studies were used as the basis for model development. The simulations carried out demonstrate when cumulative doses should be avoided. For safety, it may be desirable to consider designing a dispenser which does not allow rapid administration of more than three doses.

In carrying out this study, use of the visually oriented platform STELLA allowed ideas to be rapidly formulated for simulation and testing. 


\section{REFERENCES}

Barnes, P. J. and N. B. Pride. 1983. Dose response curves to inhaled B-adrenoreceptor agonists in normal and asthmatic subjects. British Journal of Clinical Pharmacology 15: 677-682.

Berger, R. and D. Smith. 1988. Acute bronchodilator changes in pulmonary function parameters in patients with chronic airway obstruction. Chest 93: 541-546.

Bogen, D. K. 1989. Simulation Software for the Macintosh. Science 246: 138-142.

Boyars, M. C. 1988. COPD in the ambulatory elderly: management update. Geriatrics 43: 29-40.

Hendeles, L., R. Beaty, E. Harman, L. Molino, and M. Katzper. 1992. The dose- response of albuterol during acute attacks of nocturnal asthma. First National Asthma Conference, Crystal City, Virginia.

Katzper, M. 1992. The Use of Visual Programming for Pharmacokinetic and Pharmacodynamic Simulation. Proceedings of the SCS Multiconference on Simulation in Health Care.

Lane, D. J. and A. Storr. 1979. Asthma, The Facts. Oxford University Press.

Light R. W., S. A. Conrad, and R. B. George. 1977. The one best test for evaluating effects of bronchodilator therapy. Chest 72: 512-516.

Walker S. R., M. E. Evans, A. J. Richards and J. W. Paterson. 1972. The clinical pharmacology of oral and inhaled salbutamol. Clinical Pharmacology and Therapeutics 13: 861-867.

\section{AUTHOR BIOGRAPHY}

DR. MEYER KATZPER is a Senior Research Analyst in the Research And Methodology Planning division of the Center for Drug Evaluation and Research (CDER) of the Food and Drug Administration. $\mathrm{He}$ is actively engaged in simulation, modeling and data analysis with an emphasis on pharmacokinetic and pharmacodynamic studies and data exploration. As an independent researcher and academic, Dr. Katzper has carried out studies in the areas of health, energy, environment, biophysics, physiology, social sciences and management. 